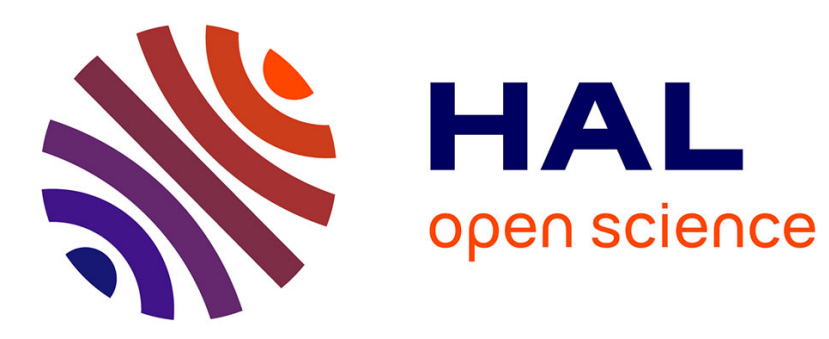

\title{
Modeling holes and inclusions by level sets in the extended finite-element method
}

Natarajan Sukumar, David Chopp, Nicolas Moës, Ted Belytschko

\section{To cite this version:}

Natarajan Sukumar, David Chopp, Nicolas Moës, Ted Belytschko. Modeling holes and inclusions by level sets in the extended finite-element method. Computer Methods in Applied Mechanics and Engineering, 2001, 190 (46-47), pp.6183-6200. 10.1016/S0045-7825(01)00215-8 . hal-01528265

\section{HAL Id: hal-01528265 https://hal.science/hal-01528265}

Submitted on 28 May 2017

HAL is a multi-disciplinary open access archive for the deposit and dissemination of scientific research documents, whether they are published or not. The documents may come from teaching and research institutions in France or abroad, or from public or private research centers.
L'archive ouverte pluridisciplinaire HAL, est destinée au dépôt et à la diffusion de documents scientifiques de niveau recherche, publiés ou non, émanant des établissements d'enseignement et de recherche français ou étrangers, des laboratoires publics ou privés.

\section{(c)(1)}

Distributed under a Creative Commons Attribution| 4.0 International License 


\title{
Modeling holes and inclusions by level sets in the extended finite-element method
}

\author{
N. Sukumar ${ }^{\mathrm{a}, *}$, D.L. Chopp ${ }^{\mathrm{b}}$, N. Moës ${ }^{\mathrm{c}}$, T. Belytschko $^{\mathrm{c}}$ \\ a Department of Civil Engineering, Northwestern University, Evanston, IL 60208, USA \\ ${ }^{\mathrm{b}}$ Department of Engineering Sciences and Applied Mathematics, Northwestern University, Evanston, IL 60208, USA \\ ${ }^{\mathrm{c}}$ Department of Mechanical Engineering, Northwestern University, Evanston, IL 60208, USA
}

\begin{abstract}
A methodology to model arbitrary holes and material interfaces (inclusions) without meshing the internal boundaries is proposed. The numerical method couples the level set method (S. Osher, J.A. Sethian, J. Comput. Phys. 79 (1) (1988) 12) to the extended finiteelement method (X-FEM) (N. Moës, J. Dolbow, T. Belytschko, Int. J. Numer. Methods Engrg. 46 (1) (1999) 131). In the X-FEM, the finite-element approximation is enriched by additional functions through the notion of partition of unity. The level set method is used for representing the location of holes and material interfaces, and in addition, the level set function is used to develop the local enrichment for material interfaces. Numerical examples in two-dimensional linear elastostatics are presented to demonstrate the accuracy and potential of the new technique. (C) 2001 Elsevier Science B.V. All rights reserved.
\end{abstract}

Keywords: Extended finite-element method; Level set method; Voids; Material interface; Elastic inclusion

\section{Introduction}

Defects such as pores, cracks, and inclusions are important to the structural integrity and durability of components. For example, material interfaces in composites are modeled to predict the mechanical behavior and to establish macroscopic material properties. The accurate modeling of voids and inclusions is hence of interest to both the theorist and the practitioner. In finite-element methods, the mesh is required to conform to internal boundaries in the model. Although mesh generation is very well established in two dimensions, meshing arbitrary number and distribution of defects and inclusions is still a time-consuming and burdensome task.

In this paper, we describe the modeling of internal boundaries from a different viewpoint, by using the level set method within the framework of the extended finite-element method (X-FEM) [8]. In the X-FEM, the finite-element approximation is enriched by additional functions through the notion of partition of unity [7]. The recognition and use of partition of unity enrichment strategy to solve boundary-value problems with internal boundaries is due to Oden and co-workers $[4,5,9,10]$ - the numerical technique was coined as the generalized finite-element method (GFEM). Strouboulis et al. [14] used local enrichment functions in the GFEM for modeling re-entrant corners in two dimensions, and in [15], enrichment functions for holes are proposed. 
In the X-FEM, the additional functions are used to model cracks, voids or inhomogeneities, and also to improve accuracy in problems where some aspects of the functional behavior of the solution field are known a priori. As opposed to the GFEM in which the analytical solution of a given boundary-value problem is embedded into the finite-element space, in the X-FEM the proximity to the finite-element method is consistently sought by carrying out only the requisite enrichment to model the internal boundary (crack or inclusion) of interest. The GFEM is amenable to accurate numerical solutions with coarse meshes and provides the capability of $p$-type adaptivity. In the X-FEM, less dependence on known closed-form solutions is emphasized which provides for greater flexibility to solve a wider range of problems. For a few applications in the above spirit, see [3,8] for crack modeling in two dimensions, and [16,17] for threedimensional elastostatics and fatigue crack growth modeling.

The level set method is a numerical technique for tracking moving interfaces [11]. The evolving interface is represented as the zero level set of a function of one higher dimension (i.e., $\varphi(\mathbf{x}, t)=0$ ). The motion of the interface is embedded in a hyperbolic equation in terms of $\varphi(\mathbf{x}, t)$. In this paper we pursue twodimensional elastostatic applications, and hence the interface is static. The interface for geometric entities such as holes and inclusions is represented by the level set function. In addition, using $\varphi$, the local enrichment function for material interfaces is constructed.

The outline of this paper is as follows. In the next section, we give a brief introduction to the X-FEM, and in Section 3, the level set method is introduced. In Section 4, the modeling of voids and inclusions is addressed. The strong and weak forms of the Galerkin method for two-dimensional elastostatics, along with the discrete equations for the X-FEM are given in Section 5. In Section 6, two different approaches for the numerical integration of the weak form are described. Numerical results are presented in Section 7, and we close with some concluding remarks in Section 8.

\section{The X-FEM}

In the finite-element method, the presence of flaws or inhomogeneities such as cracks, voids, and inclusions must be taken into account in the mesh generation process - the edges of the element must conform to these geometric entities.

The X-FEM aims to alleviate much of the burden associated with mesh generation for problems with voids and interfaces by not requiring the finite-element mesh to conform to internal boundaries. The essence of the X-FEM lies in sub-dividing a model into two distinct parts: mesh generation for the domain (excluding internal boundaries); and enriching the finite-element approximation by additional functions that model the internal boundaries.

Consider a domain $\Omega \subset \mathbb{R}^{2}$ which is partitioned into finite elements, and let $\mathbf{N}=\left\{n_{1}, n_{2}, \ldots, n_{m}\right\}$ be a set of $m$ nodes in the mesh. In addition, let $\Omega_{g} \subset \Omega$ be a region for which some enrichment is required. For a material interface, the domain $\Omega_{g}$ is the curve representing the material interface and in the case of a void, $\Omega_{g}$ is the region occupied by the void. The X-FEM displacement approximation assumes the general form:

$$
\mathbf{u}^{h}(\mathbf{x})=\sum_{\substack{I \\ n_{I} \in \mathbf{N}}} \phi_{I}(\mathbf{x}) \mathbf{u}_{I}+\sum_{\substack{J \\ n_{J} \in \mathbf{N}^{g}}} \phi_{J}(\mathbf{x}) \psi(\mathbf{x}) \mathbf{a}_{J} \quad\left(\mathbf{u}_{I}, \mathbf{a}_{J} \in \mathbb{R}^{2}\right),
$$

where the nodal set $\mathbf{N}^{g}$ is defined as

$$
\mathbf{N}^{g}=\left\{n_{J}: n_{J} \in \mathbf{N}, \omega_{J} \cap \Omega_{g}=\emptyset\right\} .
$$

In the above equation, $\omega_{J}=\operatorname{supp}\left(n_{J}\right)$ is the support of the nodal shape function $\phi_{J}(\mathbf{x})$, which consists of the union of all elements with $n_{J}$ as one of its vertices. The choice of the enrichment function $\psi(\mathbf{x})$ for voids and material interfaces will be described in Section 4. 


\section{The level set method}

The level set method [11] is a numerical technique for tracking moving interfaces. It is based upon the idea of representing the interface as a level set curve of a higher-dimensional function $\varphi(\mathbf{x}, t)$. In keeping with the applications pursued in this paper, we consider one-dimensional curves in $\mathbb{R}^{2}$.

A moving interface $\Gamma(t) \subset \mathbb{R}^{2}$ can be formulated as the level set curve of a function $\varphi: \mathbb{R}^{2} \times \mathbb{R} \rightarrow \mathbb{R}$, where

$$
\Gamma(t)=\left\{\mathbf{x} \in \mathbb{R}^{2}: \varphi(\mathbf{x}, t)=0\right\} .
$$

One important example of such a function $\varphi$ would be the signed distance function

$$
\varphi(\mathbf{x}, t)= \pm \min _{\mathbf{x}_{\Gamma} \in \Gamma(t)}\left\|\mathbf{x}-\mathbf{x}_{\Gamma}\right\|
$$

where the sign is positive (negative) if $\mathbf{x}$ is outside (inside) the contour defined by $\Gamma(t)$ (we assume that the interface $\Gamma(t)$ is such that one can define an interior and exterior to it).

The evolution of the interface is then embedded in the evolution equation for $\varphi$, which is given by Osher and Sethian [11]

$$
\begin{aligned}
& \varphi_{t}+F\|\nabla \varphi\|=0, \\
& \varphi(\mathbf{x}, 0) \text { given, }
\end{aligned}
$$

where $F(\mathbf{x}, t)$ is the speed of the interface at $\mathbf{x} \in \Gamma(t)$ in the direction of the outward normal to the interface. The key advantages of this method are that it is computed on a fixed Eulerian mesh, handles topological changes in the interface naturally, and can easily be formulated in higher dimensions. Application of the level set method to various problems in engineering and the applied sciences can be found in [13].

For our purposes in this paper, the interface is static, so we only use the level set theory for representing the interface. For circular voids, we use

$$
\varphi(\mathbf{x}, 0)=\min _{\substack{\mathbf{x}_{\mathrm{c}}^{i} \in \Omega_{\mathrm{c}}^{i} \\ i=1,2, \ldots, n_{\mathrm{c}}}}\left\{\left\|\mathbf{x}-\mathbf{x}_{\mathrm{c}}^{i}\right\|-r_{\mathrm{c}}^{i}\right\}
$$

where $\Omega_{\mathrm{c}}^{i}$ is the domain of the $i$ th void, $n_{\mathrm{c}}$ is the number of circular voids, and $\mathbf{x}_{\mathrm{c}}^{i}$ and $r_{\mathrm{c}}^{i}$ are the center and radius of the $i$ th void, respectively.

Consider an elliptical void with semi-major and semi-minor lengths of $a$ and $b$, respectively, with the axes oriented along the local $\hat{x}_{1}$ and $\hat{x}_{2}$ axes, respectively. In addition, let $\hat{\mathbf{e}}=\mathbf{R e}$ be the mapping between the global and the local unit base vectors. Now, the coordinates of a point in the local orthogonal coordinate system with the origin at the center of the ellipse is given by: $\hat{\mathbf{x}}=\mathbf{R}\left(\mathbf{x}-\mathbf{x}_{\mathrm{c}}\right)$, where $\mathbf{x}_{\mathrm{c}}$ is the center of the ellipse. Letting $\xi=\left(\xi_{1}, \xi_{2}\right)$, and $\xi_{1}=\hat{x}_{1} / a$ and $\xi_{2}=\hat{x}_{2} / b$, the equation of the ellipse in the local coordinate system is: $f(\xi)=\|\xi\|-1=0$. Hence a level set function for elliptical voids is given by

$$
\begin{aligned}
& \Phi(\mathbf{x}, 0)=\min _{i=1,2, \ldots, n_{\mathrm{e}}} f\left(\xi^{i}\right), \\
& f\left(\xi^{i}\right)=\left\|\xi^{i}\right\|-1, \\
& \xi^{i}=\left(\frac{\hat{x}_{1}^{i}}{a_{i}}, \frac{\hat{x}_{2}^{i}}{b_{i}}\right), \\
& \hat{\mathbf{x}}^{i}=\mathbf{R}^{i}\left(\mathbf{x}-\mathbf{x}_{\mathrm{c}}^{i}\right),
\end{aligned}
$$

where $n_{\mathrm{e}}$ is the number of elliptical voids. Note that in Eq. (7a), $\Phi$ is a level set function, but not a signed distance function. In order to obtain the signed distance function $\varphi$ to an elliptical void (interface), one applies the fast marching method to Eq. (9) with $G(\mathbf{x})=1$. 
Now, consider a polygonal void, whose interface $\Gamma_{p}\left(\Gamma_{p}=\bigcup_{i=1}^{p} \mathbf{l}_{i}\right)$ consists of $p$ segments: $\mathbf{l}_{1}=\left[\mathbf{x}_{1}, \mathbf{x}_{2}\right]$, $\mathbf{l}_{2}=\left[\mathbf{x}_{2}, \mathbf{x}_{3}\right], \ldots, \mathbf{l}_{p}=\left[\mathbf{x}_{p}, \mathbf{x}_{1}\right]$. The level set function for a polygonal interface is given by

$$
\begin{aligned}
& \varphi(\mathbf{x}, 0)=\left\|\mathbf{x}-\mathbf{x}_{\min }\right\| \operatorname{sgn}\left(\left(\mathbf{x}-\mathbf{x}_{\min }\right) \cdot \mathbf{n}_{\min }\right), \\
& \operatorname{sgn}(\xi)= \begin{cases}1 & \text { if } \xi \geqslant 0, \\
-1 & \text { if } \xi<0,\end{cases} \\
& \left\|\mathbf{x}-\mathbf{x}_{\min }\right\|=\min _{\substack{\mathbf{x}_{i} \in \mathbf{I}_{i} \\
i=1,2, \ldots, p}}\left\|\mathbf{x}-\mathbf{x}_{i}\right\|,
\end{aligned}
$$

where $\mathbf{x}_{\min }$ is the orthogonal projection of $\mathbf{x}$ on the interface $\Gamma_{p}$ and $\mathbf{n}_{\min }$ is the outward normal to the interface at $\mathbf{x}_{\min }$. If no unique normal is defined at $\mathbf{x}_{\min }$, the sign is positive if $\mathbf{x}-\mathbf{x}_{\min }$ belongs to the cone of normals at $\mathbf{x}_{\min }$ and negative otherwise.

In Fig. 1, the level set function for a hexagonal interface is shown. For more general interfaces given only as a parameterized curve, the signed distance function is most efficiently calculated using the fast marching method [12], which optimally solves an equation of the form

$$
\|\nabla \varphi\|=\frac{1}{G(\mathbf{x})},
$$

where $G: \mathbb{R}^{2} \rightarrow \mathbb{R}$ is given. If we take $G(\mathbf{x})=1$ and apply the fast marching method to Eq. (9), we can obtain the signed distance function to the interface. This method can be applied to both rectilinear meshes [13] and also to general triangulated meshes [1].

\section{Modeling voids and inclusions}

Level set methods are used to track the evolution of material interfaces. The geometric description of an interface (for instance, boundary of a hole or inclusion) is represented by the zero level set curve $\varphi \equiv \varphi(\mathbf{x}, t)=0$. In essence, the physical description of the interface is converted to a discrete functional representation through the function $\varphi(\mathbf{x}, t)$. The geometrical degrees of freedom at a fixed set of points $\mathbf{x}_{I}$ (nodes) are used to determine $\varphi$, and hence the location of the interface. In this study, each finite-element node is associated with a geometrical degree of freedom for the level set function. Finite-element interpolation is used to compute $\varphi$ at any point $\mathbf{x}$ in the domain

$$
\varphi(\mathbf{x})=\sum_{I} \phi_{I}(\mathbf{x}) \varphi_{I}
$$

where the summation is over all nodes in the connectivity of the element that contains $\mathbf{x}, \phi_{I}(\mathbf{x})$ are standard finite-element shape functions, and $\varphi_{I}$ are the nodal values of the level set function. The internal boundaries are then approximately piecewise linear for bilinear quadrilateral elements and exactly linear for threenoded triangular elements.

In the X-FEM, there is no need to mesh internal boundaries. The computational geometry issues that are associated with the finite-element mesh and the internal geometric entities (such as cracks or inclusions) are an important consideration in the X-FEM computations. In light of this, the level set method is an appealing choice that would greatly simplify and speed-up the geometric computations in the X-FEM. In addition, the level set function can also be used to construct enrichment functions within the X-FEM framework. This provides the motivation and seemingly natural choice to use the level set method in conjunction with the X-FEM.

\subsection{Voids}

The modeling of voids in the X-FEM is carried out using an enrichment function $V(\mathbf{x})$ for nodes that intersect the boundary of the void. If the node lies outside the void $V(\mathbf{x})=1$, and $V(\mathbf{x})=0$ if the node is in the interior of the void. In [2], the discretization of the boundary of the hole was independent of the 

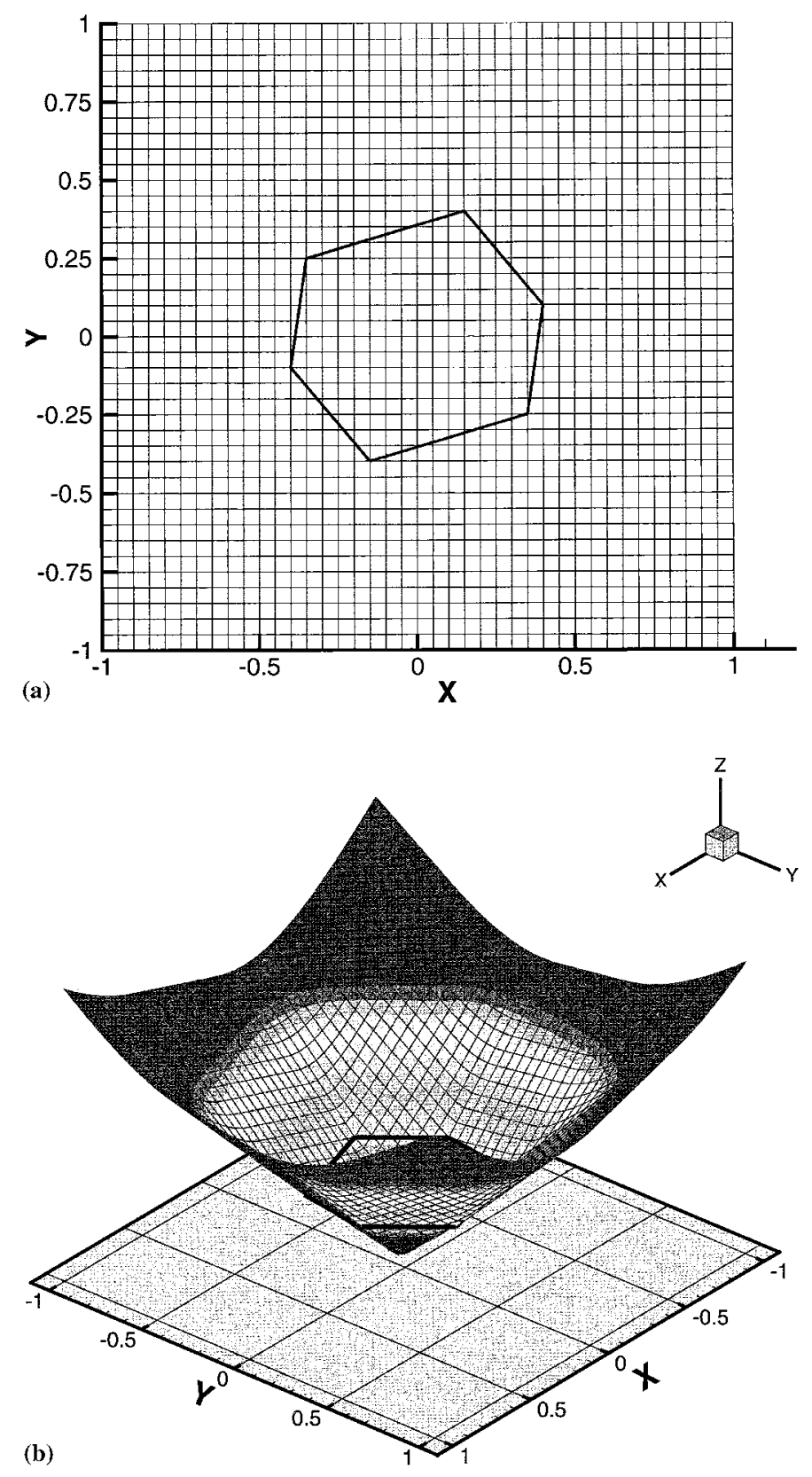

Fig. 1. Level set function for a hexagonal interface: (a) mesh; (b) level set function.

finite-element mesh. The degrees of freedom associated with nodes whose support is entirely inside the void are removed from the system of equations. In the two-dimensional implementation, instead of using $V(\mathbf{x})$, elements that intersect the boundary of the hole are partitioned such that the weak form is not integrated in the void interior [2].

In this work, the point of departure in the modeling of voids lies in using the level set method to represent the voids, and all geometric computations that pertain to the void are carried out using the level set function $\varphi$. The level set method provides a convenient and appealing means to model voids with greater flexibility and better computational efficiency. In addition, the finite-element mesh and the internal geometric boundary are linked, whereby a self-consistent representation of the internal boundary is obtained for a given finite-element mesh. 
The computation of the level set function for voids is described in Section 3. For circular voids, the geometrical degrees of freedom indicated in Eq. (10) are evaluated using Eq. (6)

$$
\varphi_{I}=\min _{\substack{\mathbf{x}_{\mathrm{c}}^{i} \in \Omega_{\mathrm{c}}^{i} \\ i=1,2, \ldots, n_{\mathrm{c}}}}\left\{\left\|\mathbf{x}_{I}-\mathbf{x}_{\mathrm{c}}^{i}\right\|-r_{\mathrm{c}}^{i}\right\}
$$

\subsection{Inclusions}

Inclusions are inhomogeneities in a matrix with differing material properties. The modeling of inclusions requires the satisfaction of the Hadamard condition, namely

$$
\mathbf{F}^{+}-\mathbf{F}^{-}=\mathbf{a} \otimes \mathbf{n}^{+}
$$

where $\mathbf{F}$ is the deformation gradient, $\mathbf{n}^{+}$is the outward normal to the material interface, and $\mathbf{a}$ is an arbitrary vector in the plane. In [6], a technique for incorporating discontinuities in derivatives for meshless methods was proposed. The use of the level set function $\varphi$ in the X-FEM provides an elegant and simplified means to achieve the same end. Using $\psi(\varphi)$ as the local enrichment, the X-FEM displacement approximation for two-dimensional domains with inclusions assumes the form:

$$
\mathbf{u}^{h}(\mathbf{x})=\sum_{\substack{I \\ n_{I} \in \mathbf{N}}} \phi_{I}(\mathbf{x}) \mathbf{u}_{I}+\sum_{\substack{J \\ n_{J} \in \mathbf{N}_{I}}} \phi_{J}(\mathbf{x}) \psi(\varphi(\mathbf{x})) \mathbf{a}_{J} \quad\left(\mathbf{u}_{I}, \mathbf{a}_{J} \in \mathbb{R}^{2}\right),
$$

where the nodal set $\mathbf{N}_{\mathrm{I}}$ is defined as

$$
\mathbf{N}_{\mathrm{I}}=\left\{n_{J}: n_{J} \in \mathbf{N}, \omega_{J} \cap \Gamma_{\mathrm{I}}=\emptyset\right\},
$$

where $\Gamma_{\mathrm{I}}$ is the boundary of the inclusion (material interface), and the nodal set $\mathbf{N}$ is defined in Section 2. The enrichment function $\psi(\varphi(\mathbf{x}))=|\varphi(\mathbf{x})|$ is used in the elements that intersect the material interface, and it is smoothed away from the interface. By construction, since $\varphi_{, n} \neq 0$, the displacement derivatives $u_{i, n}$ are discontinuous across $\Gamma_{\mathrm{I}}$.

The level set functions for inclusions follows that which is described in Section 3 for voids. We associate a level set function $\varphi$ with every distinct material-pair interface $(A-B)$. Hence, all inclusions with interfacepair $A_{\mathrm{I}}-B_{\mathrm{I}}$ are represented by the same level set function, and the number of distinct level set functions is equal to the number of distinct material-pair interfaces. The geometrical degrees of freedom $\varphi_{I}$ for circular inclusions are evaluated analogous to that given in Eq. (11).

\subsubsection{Nodal enrichment}

The level set function $\varphi$ is used to develop the local enrichment function for material interfaces. In order to determine the nodes that need to be enriched, the level set function $\varphi$ is used. We first loop over the elements of the mesh. For a given element, if there exists two nodes $n_{I}$ and $n_{J}$ in the connectivity of the element such that $\varphi_{I} \varphi_{J}<0$, we add the element $t$ to the set $\mathbf{T}$ of finite elements that intersect the interface. The enriched nodal set $\mathbf{N}_{\mathrm{e}}$ consists of all nodes that are in the connectivity of the elements in the set $\mathbf{T}$. The above algorithm ensures that if a node belongs to two edges that both lie on the interface, then the node is not enriched. Hence the finite-element space is obtained (no enrichment) if the interface lies precisely on element edges.

Let $\psi(\varphi)$ be the enrichment function for a material interface. A first and apparent choice for the enrichment function is: $\psi(\varphi)=|\varphi|$. A second alternative is to take $\psi(\varphi)=|\varphi|$ only in the elements that intersect the interface. Since the enrichment functions exist over the domain of support of the nodes, these enrichment functions are required to be computed one element layer on either side of the elements that intersect the material interface. If $\mathbf{N}_{\mathrm{e}}$ is the set of nodes that are enriched, and $\varphi_{I}$ are the level set function values at these nodes, then $\psi_{I}=\varphi_{I}$ at these nodes. Let $\mathbf{N}_{\psi}=\left\{n_{p+1}, n_{p+2}, \ldots, n_{q}\right\}$ be the set of nodes that belong to the adjacent element (outward and inward from the interface), such that $n_{I} \in \mathbf{N}_{\psi}$ are sorted in 
increasing value of the level set function $\varphi$. Initially, the value of $\psi_{I}$ is set to $\varphi_{I}$ for the nodes in $\mathbf{N}_{\psi}$, then we compute a new value for $\psi$ at these nodes based on the following algorithm:

- for $J=p+1$ to $J=q$

$\circ$ create the set $\mathbf{P}_{J}$, where

$$
\mathbf{P}_{J}=\left\{n_{I} \in \mathbf{N}_{\mathrm{e}} \cup \mathbf{N}_{\psi}:\left|\psi_{I}\right|<\left|\varphi_{J}\right| \text { and } n_{I}, n_{J} \text { share an edge in the mesh }\right\} .
$$

- minimize the following expression to obtain $\psi_{J}$ :

$$
\min \sum_{n_{I} \in \mathbf{P}_{J}}\left(\frac{\psi_{J}-\varphi_{I}}{L_{I}}\right)^{2},
$$

where $L_{I}$ is the distance from node $n_{I}$ to node $n_{J}$.

- end for

The solution to the minimization problem in Eq. (15) is explicitly given by

$$
\psi_{J}=\sum_{n_{I} \in \mathbf{P}_{J}} \alpha_{I} \varphi_{I}, \quad \alpha_{I}=\frac{1 / L_{I}^{2}}{\sum_{n_{K} \in \mathbf{P}_{J}} 1 / L_{K}^{2}},
$$

and hence the modified enrichment function is given by

$$
\psi(\mathbf{x}) \equiv \psi_{1}(\mathbf{x})=\left|\sum_{I} \phi_{I}(\mathbf{x}) \psi_{I}\right| .
$$

\section{Governing equations}

In this section, we present the governing equations of linear elastostatics, together with the weak form and the discrete system for the X-FEM. The case of internal boundaries is considered in the presentation.

\subsection{Strong form}

Consider a body which is described by an open bounded domain $\Omega \subset \mathbb{R}^{2}$, with boundary $\Gamma$. The boundary $\Gamma$ is composed of the sets $\Gamma_{u}, \Gamma_{t}, \Gamma_{h}^{i}$ and $\Gamma_{I}^{i}$, such that $\Gamma=\Gamma_{u} \cup \Gamma_{t} \cup_{i=1}^{m} \Gamma_{h}^{i} \cup_{i=1}^{n} \Gamma_{I}^{i}$. All the internal holes $\Gamma_{h}^{i}$ are assumed to be traction-free, and traction continuity holds along the material interfaces $\Gamma_{I}^{i}$. The field equations of elastostatics are:

$$
\begin{aligned}
& \boldsymbol{\nabla} \cdot \boldsymbol{\sigma}+\mathbf{b}=0 \text { in } \Omega, \\
& \boldsymbol{\sigma}=\mathbf{C}: \boldsymbol{\varepsilon}, \\
& \boldsymbol{\varepsilon}=\boldsymbol{\nabla}_{\mathrm{s}} \mathbf{u},
\end{aligned}
$$

where $\boldsymbol{\nabla}_{\mathrm{s}}$ is the symmetric gradient operator and $\mathbf{C}$ is the tensor of elastic moduli for a homogeneous isotropic material.

The essential and natural boundary conditions are:

$$
\begin{aligned}
& \mathbf{u}=\overline{\mathbf{u}} \quad \text { on } \Gamma_{u}, \\
& \boldsymbol{\sigma} \cdot \mathbf{n}=\overline{\mathbf{t}} \quad \text { on } \Gamma_{t}, \\
& \boldsymbol{\sigma} \cdot \mathbf{n}_{h}^{i}=0 \quad \text { on } \Gamma_{h}^{i} \quad(i=1,2, \ldots, m), \\
& \llbracket \boldsymbol{\sigma} \cdot \mathbf{n}_{I}^{i} \rrbracket=0 \quad \text { on } \Gamma_{I}^{i} \quad(i=1,2, \ldots, n),
\end{aligned}
$$

where $\mathbf{n}$ is the unit outward normal to $\Omega$, and $\overline{\mathbf{u}}$ and $\overline{\mathbf{t}}$ are prescribed displacements and tractions, respectively. 


\subsection{Weak form}

Let $\mathbf{u}$ be the displacement solution for the stated elastostatic boundary-value problem, with $\boldsymbol{\sigma}(\mathbf{u})$ the corresponding Cauchy stress tensor. Let $\mathbf{u} \in \mathbf{V}$ be the displacement trial solution, and $\mathbf{v} \in \mathbf{V}_{0}$ be any set of kinematically admissible test functions (virtual displacements). The space $\mathbf{V}=\mathbb{U}^{1}(\Omega)$ is the Sobolev space of functions with square-integrable first derivatives in $\Omega$, and $\mathbf{V}_{0}=\mathbb{T}_{0}^{1}(\Omega)$ is the Sobolev space of functions with square-integrable first derivatives in $\Omega$ and vanishing values on the essential boundary $\Gamma_{u}$. The weak form of the governing equation and associated boundary conditions can be written as

Find $\mathbf{u} \in \mathbf{V}$ such that

$$
\int_{\Omega} \boldsymbol{\sigma}(\mathbf{u}): \boldsymbol{\varepsilon}(\mathbf{v}) \mathrm{d} \Omega=\int_{\Omega} \mathbf{b} \cdot \mathbf{v} \mathrm{d} \Omega+\int_{\Gamma_{t}} \overline{\mathbf{t}} \cdot \mathbf{v} \mathrm{d} \Gamma \quad \forall \mathbf{v} \in \mathbf{V}_{0}
$$

\subsection{Discrete system}

Consider the Bubnov-Galerkin implementation for the X-FEM in two-dimensional linear elasticity. In the X-FEM, finite-dimensional subspaces $\mathbf{V}^{h} \subset \mathbf{V}$ and $\mathbf{V}_{0}^{h} \subset \mathbf{V}_{0}$ are used as the approximating trial and test spaces. The weak form for the discrete problem can be stated as:

Find $\mathbf{u}^{h} \in \mathbf{V}^{h} \subset \mathbf{V}$ such that

$$
\int_{\Omega^{h}} \boldsymbol{\sigma}\left(\mathbf{u}^{h}\right): \boldsymbol{\varepsilon}\left(\mathbf{v}^{h}\right)=\int_{\Omega^{h}} \mathbf{b} \cdot \mathbf{v}^{h} \mathrm{~d} \Omega+\int_{\Gamma_{t}^{h}} \overline{\mathbf{t}} \cdot \mathbf{v}^{h} \mathrm{~d} \Gamma \quad \forall \mathbf{v}^{h} \in \mathbf{V}_{0}^{h} \subset \mathbf{V}_{0} .
$$

In a Bubnov-Galerkin procedure, the trial functions $\mathbf{u}^{h}$ as well as the test functions $\mathbf{v}^{h}$ are represented as linear combinations of the same shape functions. The trial and test functions are:

$$
\begin{aligned}
& \mathbf{u}^{h}(\mathbf{x})=\sum_{I} \phi_{I}(\mathbf{x}) \mathbf{u}_{I}+\sum_{J} \phi_{J}(\mathbf{x}) \psi(\varphi(\mathbf{x})) \mathbf{a}_{J}, \\
& \mathbf{v}^{h}(\mathbf{x})=\sum_{I} \phi_{I}(\mathbf{x}) \mathbf{v}_{I}+\sum_{J} \phi_{J}(\mathbf{x}) \psi(\varphi(\mathbf{x})) \mathbf{b}_{J},
\end{aligned}
$$

where $\phi_{I}(\mathbf{x})$ are the finite-element shape functions, $\varphi(\mathbf{x})$ is the level set function, and $\psi(\varphi(\mathbf{x}))$ is the enrichment function for a material interface.

On substituting the trial and test functions from Eq. (22) in Eq. (21), and using the arbitrariness of nodal variations, the following discrete system of linear equations is obtained:

$$
\mathbf{K d}=\mathbf{f},
$$

where

$$
\begin{aligned}
& \mathbf{K}_{I J}=\int_{\Omega^{h}} \mathbf{B}_{I}^{T} \mathbf{C} \mathbf{B}_{J} \mathrm{~d} \Omega, \\
& \mathbf{f}_{I}=\int_{\Gamma_{t}^{h}} \hat{\phi}_{I} \overline{\mathbf{t}} \mathrm{d} \Gamma+\int_{\Omega^{h}} \hat{\phi}_{I} \mathbf{b} \mathrm{d} \Omega,
\end{aligned}
$$

where $\hat{\phi}_{I} \equiv \phi_{I}$ for a finite-element displacement degree of freedom, and $\hat{\phi}_{I} \equiv \phi_{I} \psi$ for an enriched degree of freedom. In the above equations, $\mathbf{C}$ is the constitutive matrix for an isotropic linear elastic material, and the matrix $\mathbf{B}_{I}$ is given by

$$
\mathbf{B}_{I}=\left[\begin{array}{cc}
\hat{\phi}_{I, x} & 0 \\
0 & \hat{\phi}_{I, y} \\
\hat{\phi}_{I, y} & \hat{\phi}_{I, x}
\end{array}\right] .
$$




\section{Numerical integration}

In standard finite-element methods, each finite element is associated with a material type; however, in the $\mathrm{X}$-FEM, since internal boundaries of geometric entities do not conform to the mesh, such an association does not exist a priori. Using the level set function $\varphi$, we can consider two different computational strategies to perform the numerical integration of the weak form. In the first approach, we establish the association between an element or sub-element (partitioned element) and a material type, and use Gauss quadrature rules in each element to carry out the numerical integration. Alternatively, since the material type of a point $\mathbf{x}$ in the domain is known by the sign of $\varphi(\mathbf{x})$, a quad-tree or octree sub-division in two- and three dimensions, respectively, in conjunction with Gauss quadrature, Simpson's rule, or other quadrature rules, is a possible candidate for the numerical integration.

In order to implement the first approach in two dimensions, we partition the finite elements that intersect the boundary of the hole or inclusion, into triangles. The level set function $\varphi$ is used to obtain the onedimensional segments of the interface, where each segment sub-divides a finite element $t \in \mathbf{T}$ (see Section 4.2.1) into precisely two domains. An algorithm is implemented to partition a finite element $t \in \mathbf{T}$ that is cut by a segment $[\mathbf{a}, \mathbf{b}]$, where $\mathbf{a}$ and $\mathbf{b}$ lie on the edges of $t$. The edges of the element $t$ are traversed and if for a particular edge $e$ with nodal connectivity $\left[n_{I}^{e}, n_{J}^{e}\right], \varphi_{I}^{e} \varphi_{J}^{e}<0$, then the intersection point $\mathbf{x}_{p}$ is determined by

$$
\mathbf{x}_{p}=\mathbf{x}_{I}^{e}+\xi\left(\mathbf{x}_{J}^{e}-\mathbf{x}_{I}^{e}\right), \quad \xi=-\frac{\varphi_{I}^{e}}{\varphi_{J}^{e}-\varphi_{I}^{e}} .
$$

The points $\mathbf{a}$ and $\mathbf{b}$ are found using the above relations. An illustration of the partitioning is depicted in Fig. 2 for an elliptical interface. In Fig. 2, the discretization of the elliptical interface that is realized by the level set computations is shown, along with the partitioned sub-elements on either side of the interface. If the discretized segment of an interface lies along an edge of a finite element, then the element is not partitioned. The above approach is accurate and feasible in two dimensions, since the discretization of the interface into linear segments and the partitioning of the finite elements into sub-triangles is readily performed. In three dimensions, however, the interface is represented by the union of triangles, and hence the implementation of a partitioning algorithm for the finite elements is non-trivial. In three-dimensional computations, the second approach we allude to has merit. By an octree sub-division of the finite elements that intersects the interface, and integrating on a given cell if all its vertices lie on the same side of the interface, an easy-to-implement algorithm emerges.

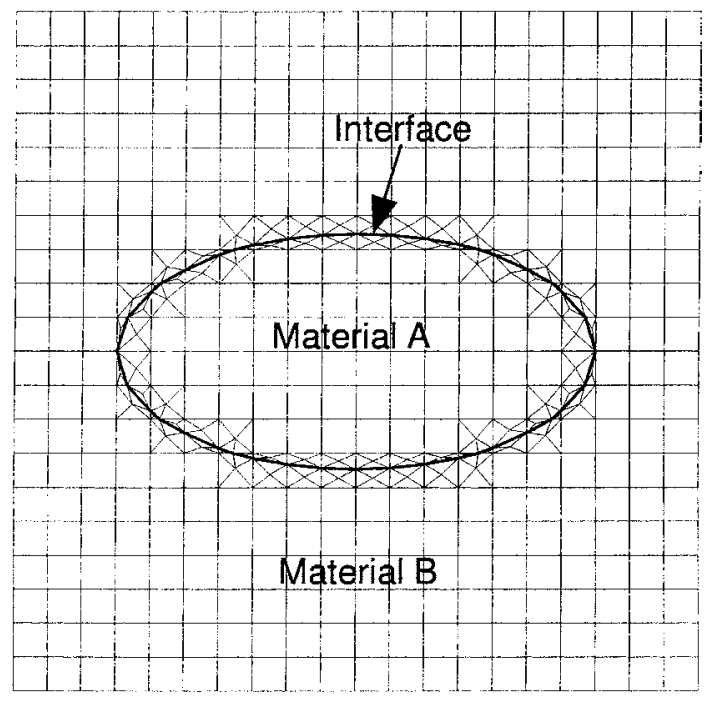

Fig. 2. Partitioning and material typing of sub-elements. 


\section{Numerical results}

Three problems in two-dimensional elastostatics are presented to illustrate the accuracy of the new technique. We first solve the problem of an infinite plate with a circular hole under uniaxial tension, and then study two problems of a bimaterial subjected to pure displacement boundary conditions. Since all problems possess an exact solution, a convergence analysis is performed to study the accuracy and rate of convergence of the proposed method. The error in the energy norm that is used in the analysis is defined as

$$
\left\|\mathbf{u}-\mathbf{u}^{h}\right\|_{E(\Omega)}=\left(\int_{\Omega}\left(\boldsymbol{\varepsilon}-\boldsymbol{\varepsilon}^{h}\right)^{\mathrm{T}} \mathbf{C}\left(\boldsymbol{\varepsilon}-\boldsymbol{\varepsilon}^{h}\right) \mathrm{d} \Omega\right)^{1 / 2} .
$$

\subsection{Infinite plate with a circular hole}

An infinite plate with a traction-free circular hole under uniaxial tension $\left(\sigma_{0}=1\right)$ along $x_{1}$ is considered. The exact solution to this problem is given in [18]. In the numerical model, we consider a square domain of edge length $L$ with a circular hole of radius $a$ at its center. We impose the exact tractions on the boundary of the square domain, with appropriate constraints added to remove rigid body modes. The material properties chosen are: Young's modulus $E=10^{5}$ and Poisson's ratio $v=0.3$.

In polar coordinates $(r, \theta)$, the exact stress distribution for $\sigma_{0}=1$ is given by

$$
\begin{aligned}
& \sigma_{11}(r, \theta)=1-\frac{a^{2}}{r^{2}}\left(\frac{3}{2}(\cos 2 \theta+\cos 4 \theta)\right) \quad \frac{3}{2} \frac{a^{4}}{r^{4}} \cos 4 \theta, \\
& \sigma_{22}(r, \theta)=-\frac{a^{2}}{r^{2}}\left(\frac{1}{2}(\cos 2 \theta-\cos 4 \theta)\right)-\frac{3}{2} \frac{a^{4}}{r^{4}} \cos 4 \theta, \\
& \sigma_{12}(r, \theta)=-\frac{a^{2}}{r^{2}}\left(\frac{1}{2}(\sin 2 \theta+\sin 4 \theta)\right) \quad \frac{3}{2} \frac{a^{4}}{r^{4}} \sin 4 \theta,
\end{aligned}
$$

where $a$ is the radius of the circular hole. The displacement components are:

$$
\begin{aligned}
& u_{1}(r, \theta)=\frac{a}{8 \mu}\left[\frac{r}{a}(\kappa+1) \cos \theta+2 \frac{a}{r}((1+\kappa) \cos \theta+\cos 3 \theta)-2 \frac{a^{3}}{r^{3}} \cos 3 \theta\right], \\
& u_{2}(r, \theta)=\frac{a}{8 \mu}\left[\frac{r}{a}(\kappa-3) \sin \theta+2 \frac{a}{r}((1-\kappa) \sin \theta+\sin 3 \theta)-2 \frac{a^{3}}{r^{3}} \sin 3 \theta\right],
\end{aligned}
$$

where $\mu$ is the shear modulus and $\kappa$ (Kolosov constant) is defined as

$$
\kappa= \begin{cases}3-4 v & \text { (plane strain) } \\ \frac{3-v}{1+v} & \text { (plane stress) }\end{cases}
$$

In the numerical computations, $L=2$ and plane strain conditions are assumed. A convergence study is carried out using equi-spaced rectangular $L_{h} \times L_{h}$ meshes: $L_{h}=10,20,40,80,160$ are used in the computations. The analyses are performed for two different hole radii: $a=0.3$ and $a=0.4$. In Fig. 3, the plot of the error in the energy norm versus the mesh spacing is shown on a log-log plot. The rate of convergence $(R)$ in the energy norm is 0.96 , which compares well to the optimal rate of one.

In order to assess the accuracy of the use of level sets in the X-FEM for modeling voids, we compare the finite-element solution to that obtained by the new technique for a mesh with approximately the same number of unknowns. The radius of the hole $a=0.4$. The finite-element mesh used 


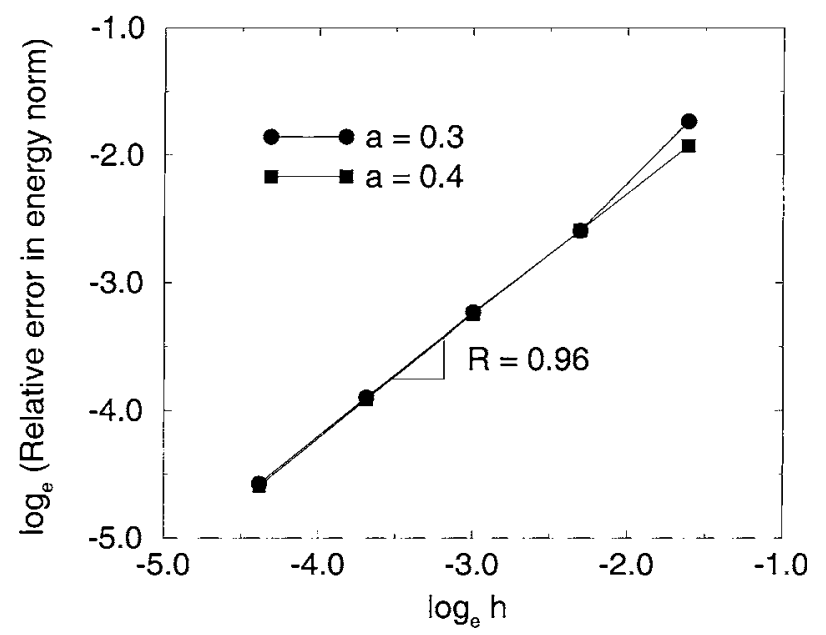

Fig. 3. Rate of convergence in energy norm for the plate with a hole problem.

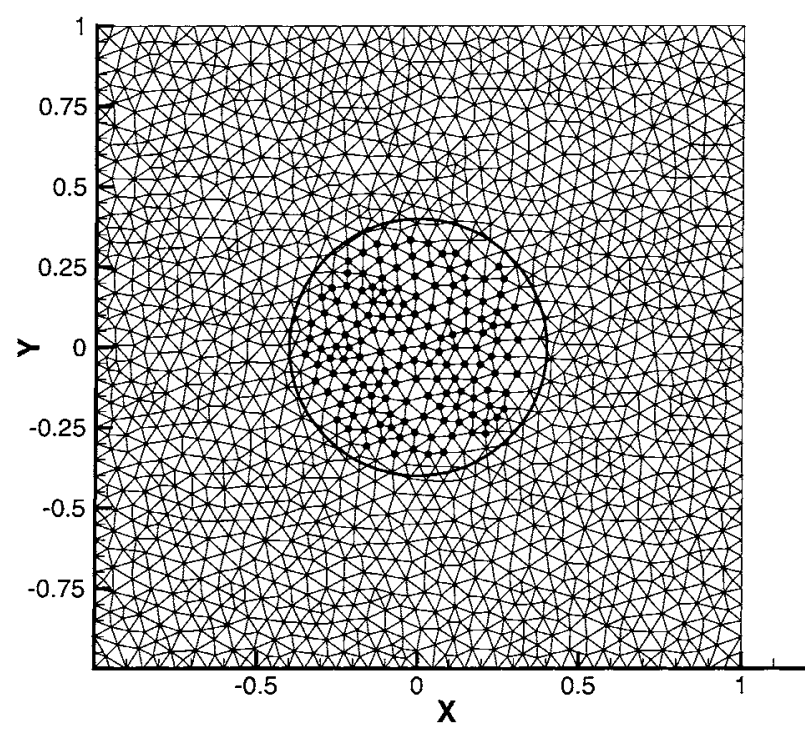

Fig. 4. Mesh used in the X-FEM computations.

consists of 3088 three-noded triangular elements with 1569 nodes (hole explicitly modeled by the mesh), and the corresponding mesh for the X-FEM implementation has 3406 three-noded triangular elements and 1704 nodes (Fig. 4). In Fig. 4, the nodes that are removed from the discrete system are indicated by the dark circles. The number of unknowns in the FE computations is 3135, and that in the $\mathrm{X}$-FEM computations is 3103 . A contour plot of the normal stress $\sigma_{11}$ using the two approaches is presented in Fig. 5. It is seen that the agreement between the results obtained by the two methods is excellent.

\subsection{Bimaterial bar}

Consider a bimaterial bar $\left(\Omega=\Omega_{1} \cup \Omega_{2}\right)$ of length $L=2$ with material moduli $E_{1}$ in $\Omega_{1}$ and $E_{2}$ in $\Omega_{2}$, where $\Omega_{1}=(-1, \xi)$ and $\Omega_{2}=(\xi, 1)$. The interface $\Gamma_{\mathrm{I}}$ is located at $x_{1}=\xi$. For the pure Dirichlet problem 

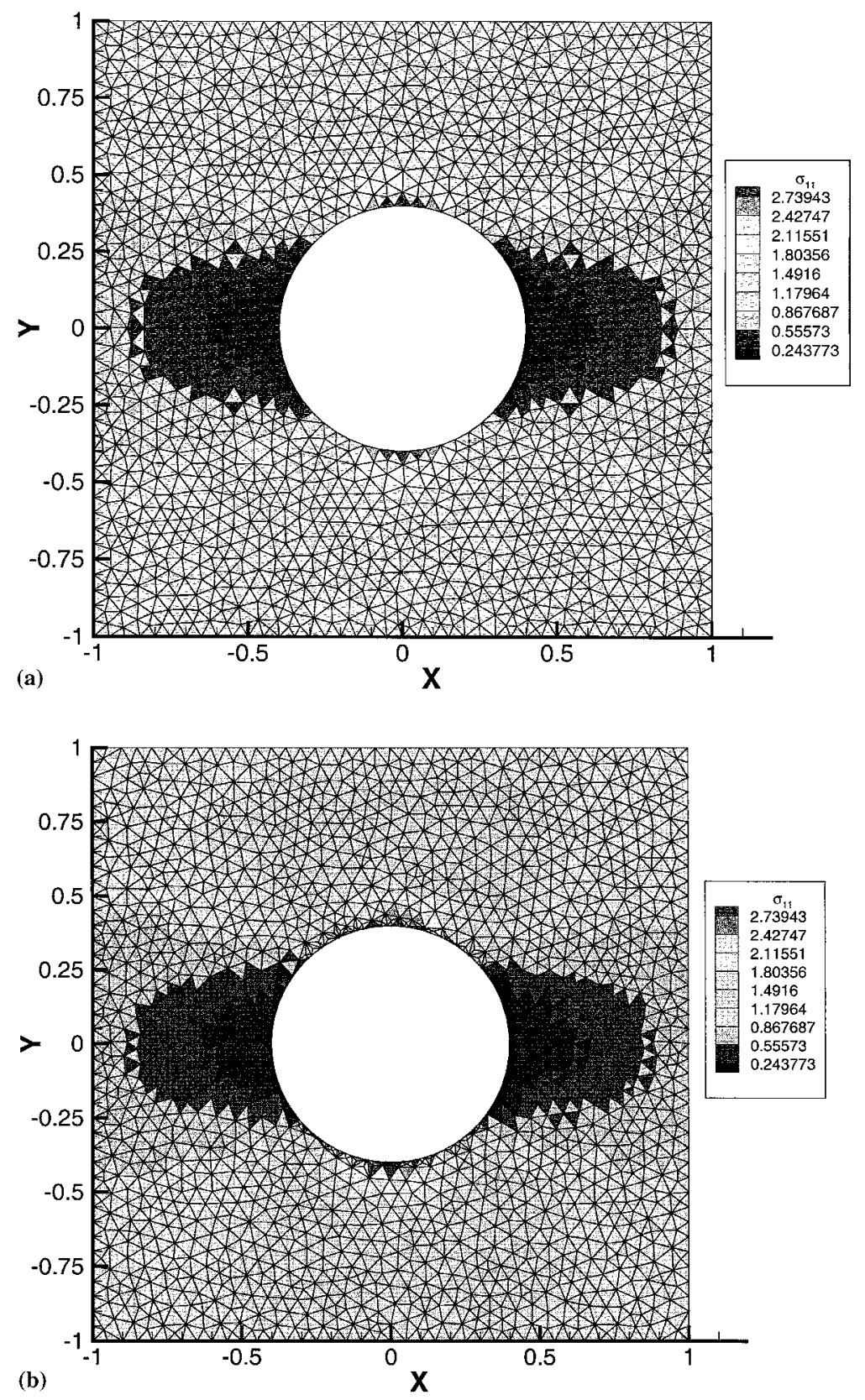

Fig. 5. Contour plot of the normal stress $\sigma_{11}$ : (a) FEM; (b) X-FEM.

with $u_{1}=0$ at $x_{1}=-1, u_{1}=1$ at $x_{1}=1$, and no body forces, the exact displacement solution assumes the form:

$$
u_{1}\left(x_{1}\right)= \begin{cases}\left(1+x_{1}\right) \alpha, & -1 \leqslant x_{1} \leqslant \xi, \\ 1+\frac{E_{1}}{E_{2}}\left(x_{1}-1\right) \alpha, & \xi \leqslant x_{1} \leqslant 1,\end{cases}
$$

where

$$
\alpha=\frac{E_{2}}{E_{2}(1+\xi)-E_{1}(\xi-1)} .
$$


In order to explore the appropriate enrichment function for the material interface, we simulate a one-dimensional bimaterial problem in a two-dimensional domain. Since the exact displacement solution is piecewise linear for the bimaterial bar in one dimension, we seek an enrichment function for the material interface that preserves the equivalence between the discrete X-FEM space and the discrete FEM space in which the interface is explicitly modeled using finite elements. By analyzing the numerical results for the bimaterial bar problem, we can generalize the enrichment methodology for general curvilinear interfaces in two dimensions, with an aim towards accuracy and convergence rate that are on par with finite elements in which the interface is explicitly modeled using elements.

We consider a two-dimensional domain $\Omega=(-1,1) \times(-1,1)$ with the material interface $\Gamma_{\mathrm{I}}$ located at $\xi \times(-1,1)$. Let Young's modulus and Poisson's ratio in $\Omega_{1}=(-1, \xi) \times(-1,1)$ be $E_{1}=1, v=0$, and that in $\Omega_{2}=(\xi, 1) \times(-1,1)$ be $E_{2}=10, v=0$. Numerical results are computed on an equi-spaced $10 \times 10$ regular finite-element mesh with five different locations of the interface: $\xi=0.01,0.05,0.1,0.15$, and 0.19 . The enrichment functions $\psi=|\varphi|$ and $\psi=\hat{\psi}$ are considered. In Fig. 6(a), the finite-element mesh and the interface are shown for $\xi=0.1$, and in Fig. $6(\mathrm{~b})$, the enrichment function $\hat{\psi}$ is illustrated. The numerical results for the relative error in the energy norm are presented in Table 1. On using $\psi=|\varphi|$ as the enrichment function, a linear approximation is not obtained in elements that are adjacent to those intersected by the interface. This adversely affects the energy error norm results. However, by using the enrichment function $\hat{\psi}$ shown in Fig. 6(b), a linear approximation is obtained in $\Omega$ which leads to very accurate results. The numerical results for this model problem indicate that the enrichment function away from the material interface needs to be a constant in order to pass the bimaterial patch test.
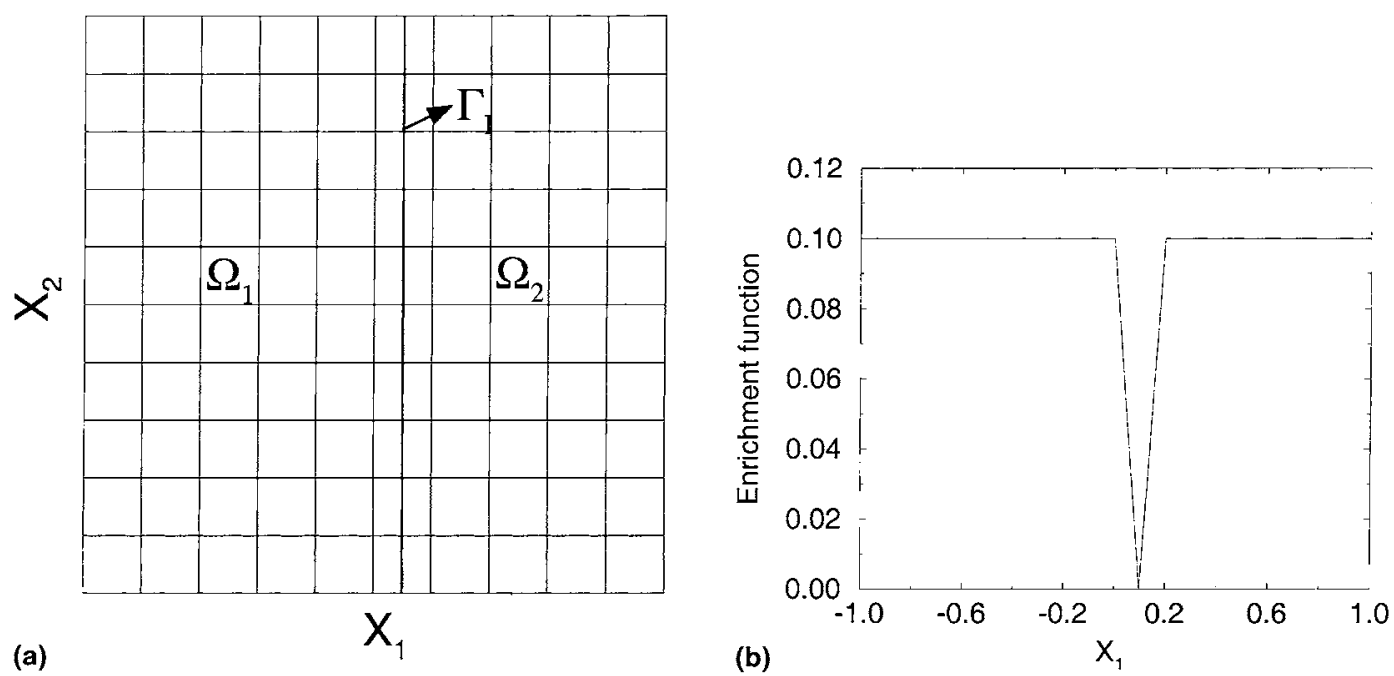

Fig. 6. Bimaterial bar problem: (a) mesh; (b) enrichment function $\hat{\psi}(\xi=0.1)$.

Table 1

Relative error in the energy norm for the bimaterial bar problem

\begin{tabular}{lll}
\hline Interface location $\xi$ & Enrichment & \\
\cline { 2 - 3 } & $|\varphi|$ & $\hat{\psi}$ \\
\hline 0.01 & $8.3 \times 10^{-2}$ & $3.0 \times 10^{-8}$ \\
0.05 & $1.6 \times 10^{-1}$ & $2.8 \times 10^{-8}$ \\
0.10 & $1.8 \times 10^{-1}$ & $2.1 \times 10^{-8}$ \\
0.15 & $1.8 \times 10^{-1}$ & $3.8 \times 10^{-8}$ \\
0.19 & $1.6 \times 10^{-1}$ & $3.6 \times 10^{-8}$ \\
\hline
\end{tabular}




\subsection{Bimaterial boundary-value problem}

In Fig. 7, a body composed of two different materials is shown. The material constants are constant in $\Omega_{1}$ and $\Omega_{2}$, but there is a discontinuity in the material constants across the interface $\Gamma_{1}(r=a)$. The Lamé constants in $\Omega_{1}$ are chosen as: $\lambda_{1}=\mu_{1}=0.4$, and those in $\Omega_{2}$ are: $\lambda_{2}=5.7692, \mu_{2}=3.8461$. These correspond to $E_{1}=1, v_{1}=0.25$, and $E_{2}=10, v_{2}=0.3$. We impose the linear displacement field: $u_{1}=x_{1}, u_{2}=x_{2}$ $\left(u_{r}=r, u_{\theta}=0\right)$ on the boundary $\Gamma_{2}(r=b)$. Navier's equation in polar coordinates reduces to

$$
\frac{\mathrm{d}}{\mathrm{d} r}\left[\frac{1}{r} \frac{\mathrm{d}}{\mathrm{d} r}\left(r u_{r}\right)\right]=0
$$

By considering displacement and traction continuity across the interface, the exact displacement solution can be written as

$$
\begin{aligned}
& u_{r}(r)= \begin{cases}{\left[\left(1-\frac{b^{2}}{a^{2}}\right) \alpha+\frac{b^{2}}{a^{2}}\right] r,} & 0 \leqslant r \leqslant a, \\
\left(r-\frac{b^{2}}{r}\right) \alpha+\frac{b^{2}}{r}, & a \leqslant r \leqslant b,\end{cases} \\
& u_{\theta}=0,
\end{aligned}
$$

where

$$
\alpha=\frac{\left(\lambda_{1}+\mu_{1}+\mu_{2}\right) b^{2}}{\left(\lambda_{2}+\mu_{2}\right) a^{2}+\left(\lambda_{1}+\mu_{1}\right)\left(b^{2}-a^{2}\right)+\mu_{2} b^{2}} .
$$

The radial $\left(\varepsilon_{r r}\right)$ and hoop $\left(\varepsilon_{\theta \theta}\right)$ strains are given by:

$$
\varepsilon_{r r}(r)= \begin{cases}\left(1-\frac{b^{2}}{a^{2}}\right) \alpha+\frac{b^{2}}{a^{2}}, & 0 \leqslant r \leqslant a, \\ \left(1+\frac{b^{2}}{r^{2}}\right) \alpha-\frac{b^{2}}{r^{2}}, & a \leqslant r \leqslant b,\end{cases}
$$

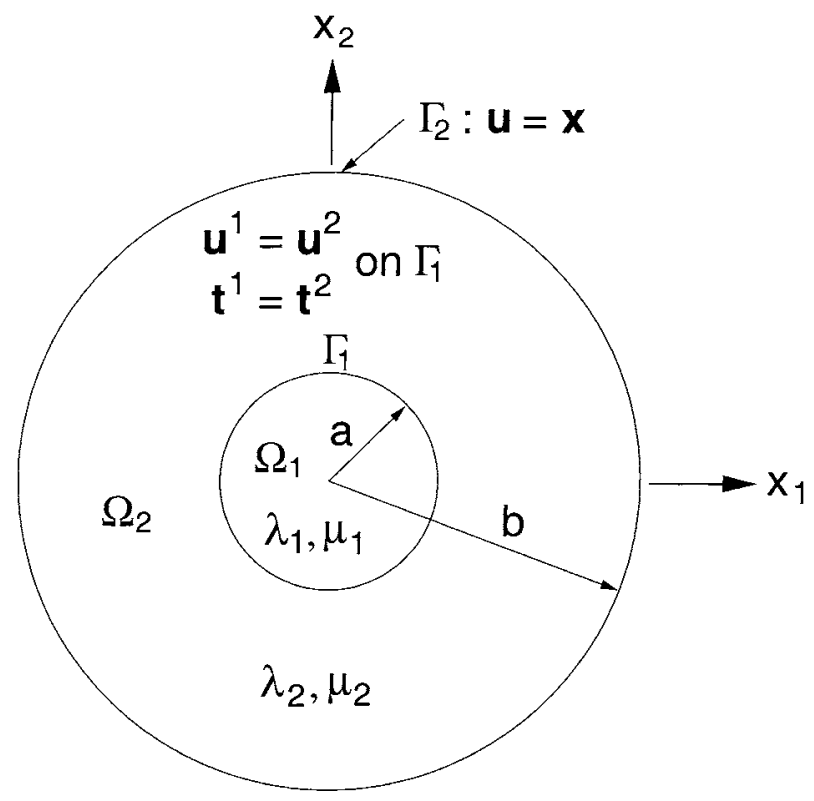

Fig. 7. Bimaterial boundary-value problem. 


$$
\varepsilon_{\theta \theta}(r)= \begin{cases}\left(1-\frac{b^{2}}{a^{2}}\right) \alpha+\frac{b^{2}}{a^{2}}, & 0 \leqslant r \leqslant a, \\ \left(1-\frac{b^{2}}{r^{2}}\right) \alpha+\frac{b^{2}}{r^{2}}, & a \leqslant r \leqslant b,\end{cases}
$$

and the radial $\left(\sigma_{r r}\right)$ and hoop $\left(\sigma_{\theta \theta}\right)$ stresses are:

$$
\begin{aligned}
& \sigma_{r r}(r)=2 \mu \varepsilon_{r r}+\lambda\left(\varepsilon_{r r}+\varepsilon_{\theta \theta}\right), \\
& \sigma_{\theta \theta}(r)=2 \mu \varepsilon_{\theta \theta}+\lambda\left(\varepsilon_{r r}+\varepsilon_{\theta \theta}\right),
\end{aligned}
$$

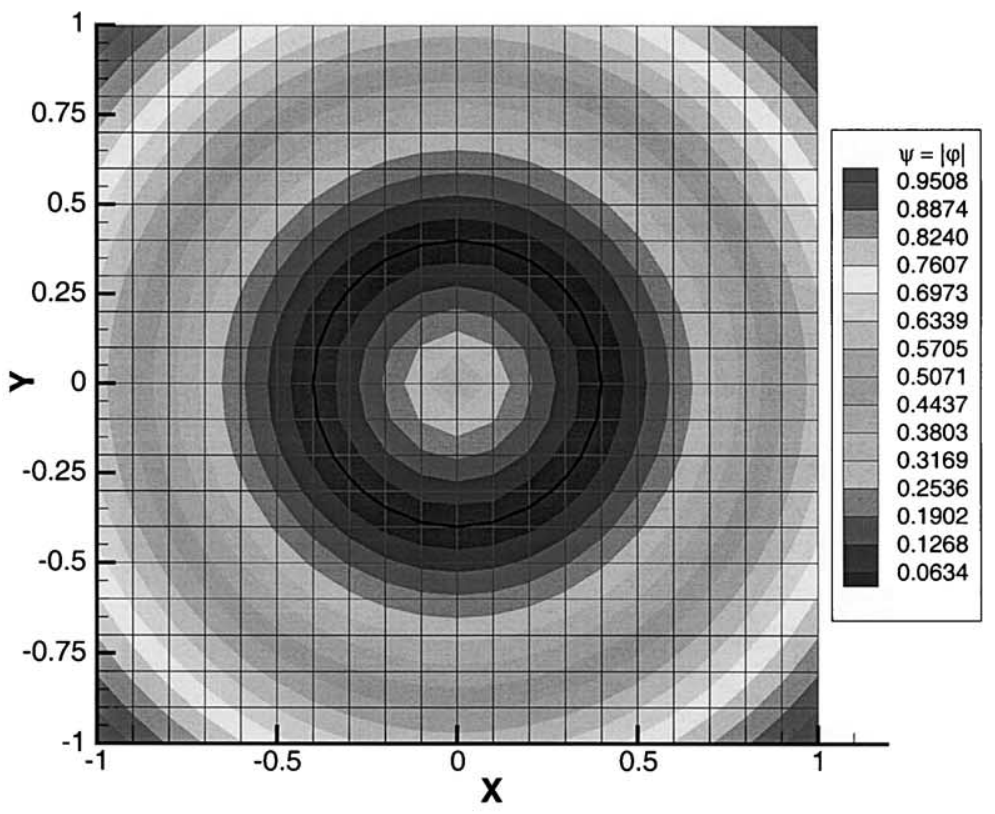

(a)

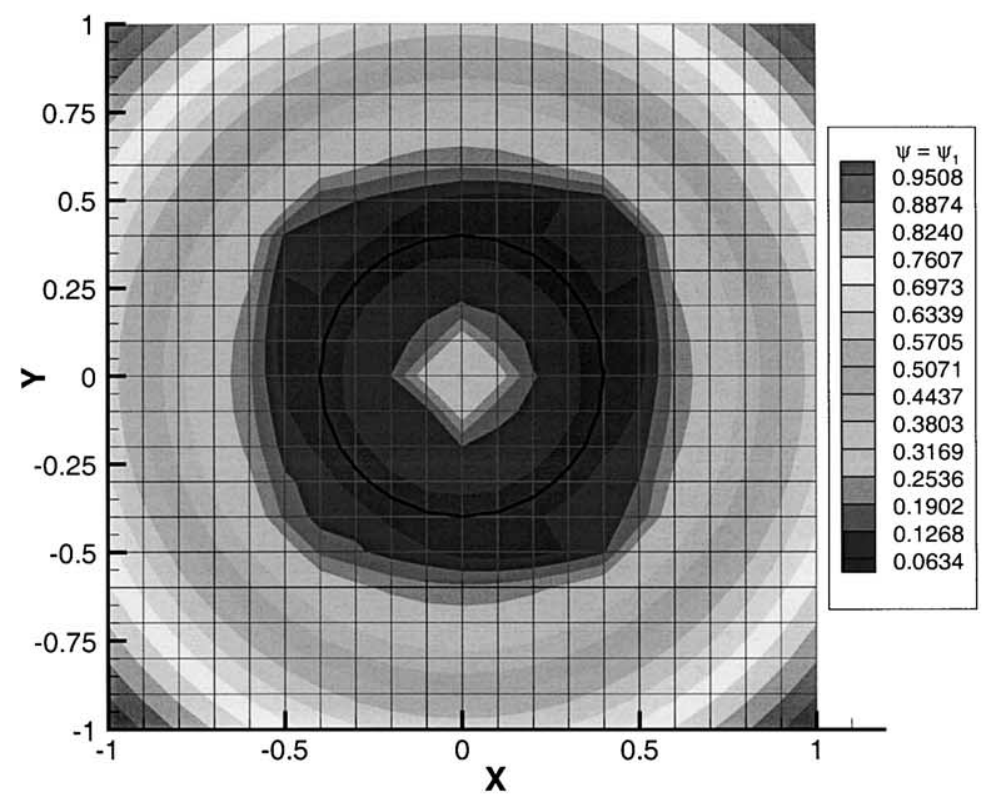

(b)

Fig. 8. X-FEM enrichment for material interfaces: (a) $\psi=|\varphi|$; (b) $\psi=\psi_{1}$. 
where the appropriate Lamé constants are to be used in the evaluation of the normal stresses. The shear components of the stress and strain tensors are zero.

In the numerical model, we consider a square plate $(L \times L, L=2)$ with a circular inclusion $\left(\Omega_{1}\right)$ of radius $a=0.4$. On the boundary of the plate, the exact tractions using Eq. (37a) are imposed $(a=0.4$ and $b=2.0$ are chosen) with appropriate displacement constraints added to remove rigid body modes. A convergence study is conducted using equi-spaced rectangular $L_{h} \times L_{h}$ meshes: $L_{h}=10,20,40,80$. The finite-element computations are carried out using three-noded triangular elements.

The convergence analysis is carried out for three cases using the X-FEM: (a) no enrichment; (b) enrichment function $\psi=|\varphi|$; and (c) enrichment function $\psi=\psi_{1}$, where $\psi_{1}$ is the modified level set function based on the smoothing procedure described in Section 4.2.1. The motivation for the smoothing procedure presented in Section 4.2.1 stems from the numerical experiments conducted in Section 7.2. The enrichment functions for the $20 \times 20$ mesh is shown in Fig. 8. In all the above cases, the weak form is integrated appropriately by partitioning the elements that are intersected by the interface. The degrees of freedom of the meshes used in the FEM and in the X-FEM computations are presented in Table 2. The numerical results for the convergence in energy norm are shown in Fig. 9. The accuracy and rate of convergence of the $\mathrm{X}$-FEM with the smoothed form of the enrichment are significantly better than the other two cases. The rate $(R=0.91)$ using the enrichment function $\psi_{1}$ is also on par with the optimal rate of unity which is obtained using finite elements with the interface truly modeled by the mesh.

Even though the enrichment function $\psi_{1}$ used in this paper is appropriate from an accuracy and convergence viewpoint, a more general enrichment methodology which would be valid for a wider class of interface problems is attractive. To this end, one can consider the enrichment nodal data $\psi_{I}$ as also unknowns in the statement of the principle of virtual work (variational form). The nodal data $\psi_{I}$ are known on nodes that are in the connectivity of the elements that intersect the interface: the modulus of the level set function $(|\varphi|)$ is an appropriate choice for this purpose. On elements that are one layer on either side (outer and inner) of these elements, the $\psi_{I}$ are to be determined by the numerical procedure. As an initial guess,

Table 2

Degrees of freedom for the meshes used for the bimaterial problem

\begin{tabular}{lrrr}
\hline Mesh $(h)$ & FEM & X-FEM (no enrichment) & X-FEM (enrichment) \\
\hline 0.2 & 277 & 239 & 287 \\
0.1 & 915 & 879 & 991 \\
0.05 & 3559 & 3359 & 3599 \\
0.025 & 13,693 & 13,119 & 13,615 \\
\hline
\end{tabular}

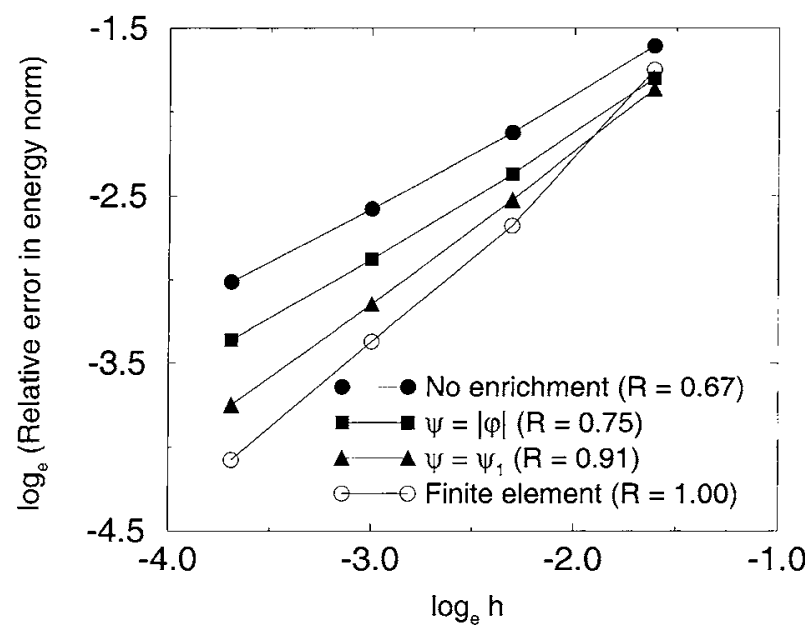

Fig. 9. Rate of convergence in energy norm for the bimaterial problem. 
the $\psi_{I}$ can be set using the smoothing procedure given in Eqs. (15)-(17). Thereafter, an iterative scheme between the solution of the minimization problem with respect to $\left\{u_{I}, a_{I}\right\}$ and that with respect to $\psi_{I}$ would provide the minimization of the global problem. This would result in an optimum choice for $\psi_{I}$, and an appropriate form for the enrichment function $\psi$ which is best suited for a given problem.

\section{Conclusions}

A methodology to model arbitrary holes and material interfaces (inclusions) without meshing the internal boundaries was proposed. The numerical method couples the level set method [11] to the X-FEM [8]. In the X-FEM, the finite-element space is enriched by adding additional functions to the approximation using the notion of partition of unity. The level set function $\varphi$ is used to represent holes and inclusions, and in addition, $\varphi$ is used to develop the local enrichment for material interfaces to model inclusions.

Numerical examples in two-dimensional linear elastostatics were presented to test the accuracy of the new technique. For the plate with a hole problem, a convergence study was performed for two different radii of the hole. Optimal rate of convergence in energy norm $(\mathcal{O}(h))$ was obtained using the new technique. A bimaterial boundary-value problem with pure displacement boundary conditions was also considered. Issues pertaining to appropriate choice of enrichment functions for material interfaces were addressed, and a suitable enrichment function was derived that yielded accurate results and near optimal rate of convergence for the two-dimensional bimaterial problem. This study demonstrated that by incorporating level set functions in the X-FEM, we obtain a powerful and accurate numerical technique for the modeling of voids and inclusions without meshing the internal boundaries. The new technique is simple and versatile, and the results indicate its potential and promise in the modeling of interfacial phenomena in solid and fluid mechanics.

\section{Acknowledgements}

The authors are grateful for the research support of the National Science Foundation through contract CMS-9732319, and to the Office of Naval Research and the Army Research Office.

\section{References}

[1] T.J. Barth, J.A. Sethian, Numerical schemes for the Hamilton-Jacobi and level set equations on triangulated domains, J. Comput. Phys. 145 (1) (1998) 1-40.

[2] C. Daux, N. Moës, J. Dolbow, N. Sukumar, T. Belytschko, Arbitrary cracks and holes with the extended finite element method, Int. J. Numer. Methods Engrg. 48 (12) (2000) 1741-1760.

[3] J. Dolbow, N. Moës, T. Belytschko, Modeling fracture in Mindlin-Reissner plates with the extended finite element method, Int. J. Solids Struct. 37 (48-50) (2000) 7161-7183.

[4] C.A. Duarte, I. Babuška, J.T. Oden, Generalized finite element methods for three dimensional structural mechanics problems, in: S.N. Atluri, P.E. O'Donoghue (Eds.), Modeling and Simulation Based Engineering: Proceedings of the International Conference on Computational Engineering Science, vol. I, Technical Science Press, Atlanta, GA, October 1998, pp. 53-58.

[5] C.A. Duarte, I. Babuška, J.T. Oden, Generalized finite element methods for three dimensional structural mechanics problems, Comput. Struct. 77 (2000) 215-232.

[6] Y. Krongauz, T. Belytschko, EFG approximation with discontinuous derivatives, Int. J. Numer. Methods Engrg. 41 (7) (1998) 1215-1233.

[7] J.M. Melenk, I. Babuška, The partition of unity finite element method: Basic theory and applications, Comput. Methods Appl. Mech. Engrg. 139 (1996) 289-314.

[8] N. Moës, J. Dolbow, T. Belytschko, A finite element method for crack growth without remeshing, Int. J. Numer. Methods Engrg. 46 (1) (1999) 131-150.

[9] J.T. Oden, C.A. Duarte, O.C. Zienkiewicz, A new cloud-based $h p$ finite element method, Technical Report TICAM Report 96-55, The University of Texas at Austin, Austin, TX, December 1996.

[10] J.T. Oden, C.A. Duarte, O.C. Zienkiewicz, A new cloud-based $h p$ finite element method, Comput. Methods Appl. Mech. Engrg. 153 (1-2) (1998) 117-126.

[11] S. Osher, J.A. Sethian, Fronts propagating with curvature-dependent speed: Algorithms based on Hamilton-Jacobi formulations, J. Comput. Phys. 79 (1) (1988) 12-49. 
[12] J.A. Sethian, A marching level set method for monotonically advancing fronts, Proc. Nat. Acad. Sci. 93 (4) (1996) $1591-1595$.

[13] J.A. Sethian, Level Set Methods and Fast Marching Methods: Evolving Interfaces in Computational Geometry, Fluid Mechanics, Computer Vision, and Materials Science, Cambridge University Press, Cambridge, UK, 1999.

[14] T. Strouboulis, I. Babuška, K. Copps, The design and analysis of the generalized finite element method, Comput. Methods Appl. Mech. Engrg. 181 (1-3) (2000) 43-69.

[15] T. Strouboulis, K. Copps, I. Babuška, The generalized finite element method: An example of its implementation and illustration of its performance, Int. J. Numer. Methods Engrg. 47 (8) (2000) 1401-1417.

[16] N. Sukumar, D.L. Chopp, B. Moran, Extended finite element method and fast marching method for three dimensional fatigue crack propagation, J. Comput. Phys. (submitted).

[17] N. Sukumar, N. Moës, B. Moran, T. Belytschko, Extended finite element method for three-dimensional crack modeling, Int. J. Numer. Methods Engrg. 48 (11) (2000) 1549-1570.

[18] B. Szabó, I. Babuška, Finite Element Analysis, Wiley, New York, 1991. 